\title{
Ensembles of Fuzzy Classifiers
}

\author{
Juana Canul-Reich, Larry Shoemaker and Lawrence O. Hall
}

\begin{abstract}
The use of bagging is explored to create an ensemble of fuzzy classifiers. The learning algorithm used was ANFIS (Adaptive Neuro-Fuzzy Inference Systems). We compare results from bagging to those of a single classifier using both crisp and fuzzy classifier combination methods. Results on 20 data sets show that bagging results in a significantly more accurate classifier.
\end{abstract}

\section{INTRODUCTION}

There have been a number of papers which show ensembles of classifiers can be used to obtain more accurate predictions on unseen data than those from a single classifier [1]. The question being examined in this paper is whether an ensemble of fuzzy classifiers will result in higher classification accuracy than using a single fuzzy classifier. Of course, using an ensemble may cause you to lose the simplicity and clarity of the fuzzy model. However, if an accuracy gain is available this may suggest there are new ways to build higher accuracy, but still understandable, fuzzy classifiers.

Our work uses ANFIS (Adaptive Neuro-Fuzzy Inference Systems), a fuzzy classifier that is part of the MATLAB Fuzzy Logic Toolbox [2]. Bagging is the ensemble approach whose accuracy is compared to that of a single ANFIS classifier. The Friedman-Holm statistical tests were performed on the results to determine whether there was a significant difference in predictive accuracy between bagging and a single classifier. This test shows that there is significant difference at the $95 \%$ level $(\alpha=0.05)$.

Most datasets used for the experiments came from the UCI repository [3]. A feature selection process was applied to some of them to make them suitable for use with ANFIS. In addition, a stratification process was performed on all data sets for class distribution preservation purposes.

The contents of the paper are organized as follows. The related work section includes a brief description of previous work regarding ensembles of classifiers. Then, a section describing ANFIS is followed by the ANFIS ensemble section, where the bagging process is described. Next comes the experimental evaluation section, which has two subsections, one describing the data set selection process and one containing a description of the experiments. Finally, an analysis of results is given followed by the conclusions.

Juana Canul-Reich is with the Department of Computer Science and Engineering, University of South Florida, 4202 E. Fowler Avenue, Tampa, FL., USA (phone: +1 813 9743033; email: jcanulre@ cse.usf.edu).

Larry Shoemaker is with the Department of Computer Science and Engineering, University of South Florida, 4202 E. Fowler Avenue, Tampa, FL., USA (phone: +1 813 9743033; email: lwshoema@cse.usf.edu).

L. O. Hall is with the Department of Computer Science and Engineering, University of South Florida, 4202 E. Fowler Avenue, Tampa, FL., USA (phone: +1 813 9744195; email: hall@cse.usf.edu).

\section{RELATED WORK}

In general, accuracy obtained from an ensemble of classifiers is expected to be higher than that obtained from a single classifier. The ensemble generation approach studied is bagging, particularly the bagging of fuzzy classifiers.

Bagging was first introduced by Breiman in [4]. Breiman implemented bagging on classification trees using moderate sized as well as larger data sets. Bagging was also applied to regression trees, linear regression and nearest neighbor classifiers. Results showed that bagging improved performance on unstable classifiers. A classifier is said to be stable when relatively small changes in the training set do not cause significant changes in the classifier itself.

ANFIS, the fuzzy classifier utilized in this work, has also been applied in another ensemble approach. In [5], the authors create ensembles by using different types of fuzzy membership functions within ANFIS. The maximum ensemble size was eight and they used a weighting algorithm to get the final output prediction. They show results on several small data sets for which a single numeric output is desired. They do get less error, but do not address the problem of classification.

A general fuzzy min-max (GFMM) neural network is described in [6]. This method can be used for pure clustering, pure classification, or a combination of both. Hyperbox fuzzy sets are used to represent clusters and classes. Learning is accomplished by placing and adjusting the hyperbox in pattern space. GFMM classification results can be crisp or fuzzy. The IRIS, wine, and ionosphere data sets were tested, and the classification performance of GFMM on the iris data set compares favorably to that of various traditional, fuzzy, and neural classifiers.

The combination of multiple GFMM neuro-fuzzy classifiers was examined in [7]. Conventional resampling methods to generate ensembles of classifiers include boosting and bagging. In these methods, multiple copies of a classifier are generated by applying the base learning algorithm to different subsets of the original training data. In contrast to combining the decisions of multiple copies of the GFMM classifier, an alternative method is proposed that combines hyperbox fuzzy sets from multiple GFMM classifier models into a single classifier model. The resulting performance and stability of the ensemble was improved without sacrificing model transparency and introducing more complexity.

\section{ANFIS}

ANFIS (Adaptive-Network-based Fuzzy Inference System) is a fuzzy inference system implemented under the framework of adaptive networks [8]. An adaptive network can be considered as a superset of feed-forward neural 
networks with supervised learning. ANFIS is a type of Neurofuzzy network which has the fuzzy rules embedded within the neural network. Figure 1 shows the structure of an adaptive network. Node functions are represented by squares if they have parameters, which make them adaptive, and by circles if they do not have parameters. The links have no associated weights and they only represent direction flow. For further details on ANFIS, see [8].

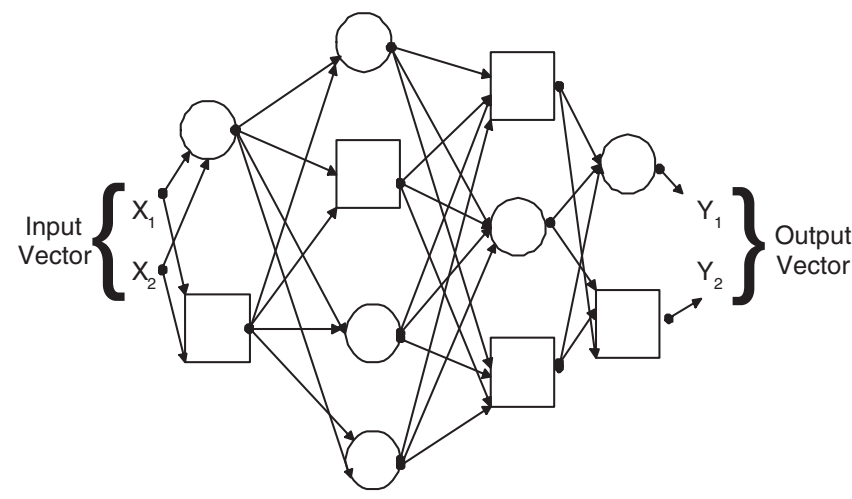

Fig. 1. Structure of an Adaptive Network

\section{ANFIS ENSEMBLE}

We applied bagging to build ensembles of ANFIS fuzzy classifiers. In bagging, $n$ bags are formed, each one is obtained via sampling the total set of instances with replacement from the training set, typically, a bag is the same size as the training set. Then, we apply a learning algorithm (ANFIS in our case) using each bag as a training set in order to obtain a separate classifier. Then each classifier predicts the class for each of the instances in the testing set. The final predicted class for each test instance may be computed by a majority vote, see [9].

\section{EXPERIMENTAL EVALUATION}

First, the reason for the selection of the data sets is reviewed. Next, a detailed description of all experiments performed is provided.

\section{A. Selecting Data Sets}

In Table I we show the twenty data sets selected for our experiments from different sources, mostly from the UCI repository [3]. ANFIS results are typically poor for datasets with more than six features due to the size of the fuzzy search space. In the data mining tool, Weka [10], the gain ratio feature selector was used to choose the best 6 features for data sets with more than six features.

\section{B. Experiments}

The ANFIS classifier was tested on twenty data sets, both without bagging and with 2 types of vote counting for the bagged ensemble. Each experiment on a data set begins with a stratified separation of the data into approximately $2 / 3$ of the examples for training, and the remaining examples (approximately 1/3) for testing. The stratification process
TABLE I

Selected Data Sets

\begin{tabular}{l|c|c|c|c}
\hline \hline \multicolumn{1}{c|}{ Data set } & $\begin{array}{c}\text { No. attributes } \\
\text { utilized }\end{array}$ & $\begin{array}{c}\text { Total } \\
\text { attributes }\end{array}$ & No. Instances & No. Classes \\
\hline balance_scale & 4 & 4 & 625 & 3 \\
breast-w & 6 & 9 & 699 & 2 \\
cmc & 6 & 9 & 1473 & 3 \\
dermatology & 6 & 34 & 366 & 6 \\
glass & 6 & 9 & 214 & 7 \\
haberman & 3 & 3 & 306 & 2 \\
heart-statlog & 6 & 13 & 270 & 2 \\
ionosphere & 6 & 34 & 351 & 2 \\
iris & 4 & 4 & 150 & 3 \\
monks1 & 6 & 6 & 432 & 2 \\
monks2 & 6 & 6 & 432 & 2 \\
newthyroid & 5 & 5 & 215 & 3 \\
page-blocks & 6 & 10 & 5473 & 5 \\
phoneme & 5 & 5 & 5404 & 2 \\
pima & 5 & 8 & 768 & 2 \\
satimage-test & 6 & 36 & 2000 & 6 \\
tae & 5 & 5 & 151 & 3 \\
vehicle & 6 & 18 & 846 & 4 \\
wine & 6 & 178 & 3 \\
yeast & 5 & 5 & & 10 \\
\hline \hline
\end{tabular}

is intended to preserve the class distribution present in the original data set for both training and testing sets. Then for each of 100 bags, a bag of data was created by randomly drawing with replacement from the stratified training set until the number of examples in the bag equals the stratified training set size. This bag probably will have numerous instances of examples that are in the bag more than once. Conversely, some of the examples in the stratified set will not be drawn and put into the bag. These out-of-bag examples are used for the checking or validation set. The checking set is used by ANFIS to prevent overfitting the training data, especially if the data has noise or if the number of training epochs is large.

In order to evaluate both methods of bagging with a single classifier that does not use bagging, one instance of each unique example in the stratified training set was used to create the training data set. This can also be viewed as simply removing all duplicates or multiple instances of examples from the bag. This method uses the same checking set used for the bagging trials, which should provide a fair comparison of bagging vs. no bagging.

ANFIS is run using the data in the newly formed training set (either a bag or that bag with duplicates removed) as an input to train the FIS (Fuzzy Inference System). A separate checking FIS is generated that captures the parameters of the training FIS in the epoch of minimum error, which results in a more accurate model. When the training process is complete, the checking FIS is used to classify the unseen test data.

The above process was repeated in each experiment for the 
number of bags we chose to use for experiments, which was 100. Each new bagged and non bagged classifier was formed from the same stratified training set that was selected from the entire data set before the first bag was formed.

When all 100 classifiers have been created, two different types of ensemble voting were performed on the outputs generated by the checking FIS for test examples. In the first type of voting, the predictions for each test example consist of the defuzzified outputs from the checking FIS. These real numbers are rounded to crisp values (whole numbers) and any resulting value that is invalid or out-of-range is changed to the closest valid class value. Then the 100 crisp votes are counted and the predicted class for the example is the one with the majority of votes. In the case of ties, the class with the lower number wins.

In the other type of ensemble voting, the 100 defuzzified votes are simply added. Then the mean or average value is determined. This value is then rounded to a valid, crisp class value and is designated as the class predicted by the ensemble. For example, consider the case of 3 classifiers predicting 0.4, 1 and 0.4 respectively for a sample (prediction $\leq 0.5$ means class 1 and prediction $>0.5$ means class 2 ). Under the majority-of-votes criteria, these individual predictions are clearly $1,2,1$, resulting the majority of votes for class 1 . Under the mean-of-defuzzified-votes criteria, the mean of the three original predictions is calculated, that is 0.6 , resulting in a combined prediction for class 2 for the sample.

The process described above was performed 25 times and average accuracies are reported.

\section{Analysis of Results}

In Table II the average test accuracies are shown for 25 test runs using the ANFIS checking FIS for 10 epochs. A visual representation of these results is shown in Figure 2.

Figure 2 shows higher accuracy was achieved with the defuzzified ensemble voting method on fifteen out of twenty test data. The worst accuracy consistently came from the majority vote bagging ensemble method.

In Table III the average test accuracies are shown for 25 test runs using the ANFIS checking FIS for 20 epochs.

Table III and Figure 3 indicate that higher accuracy was achieved on the glass and yeast data sets with bagging using the defuzzified mean.

Figure 4 shows a head-to-head comparison of 10 vs. 20 epochs for test accuracies using defuzzified voting of predictions from the checking FIS generated using bags of data. The accuracy with 20 epochs was greater than or equal to that of 10 epochs, except for iris and newthyroid datasets.

The significance of the accuracy difference between bagging and a single classifier was evaluated using the FriedmanHolm Test, which was discussed in [11]. The procedure allows the comparison of two or more classifiers over multiple data sets and determines whether there is a statistically significant difference in the accuracies. It uses the ranks of the classifier on each data set, ranging from 1-3 here. Ties of 1 , for example, are each given 1.5 , and smaller is better.
TABLE II

AVERAGE TEST ACCURACIES IN \% FOR 25 RUNS USING CHECKING FIS FOR 10 EPOCHS. A BOLD VALUE INDICATES THE HIGHEST ACCURACY FOR THAT DATA SET.

\begin{tabular}{l|c|c|c}
\hline \hline \multicolumn{1}{c|}{ Data set } & no bags & $\begin{array}{c}\text { bags } \\
\text { majority vote }\end{array}$ & $\begin{array}{c}\text { bags } \\
\text { mean defuzzified }\end{array}$ \\
\hline balance_scale & 71.229 & 69.627 & $\mathbf{7 1 . 5 2 2}$ \\
breast & 92.769 & 81.236 & $\mathbf{9 3 . 5 7 9}$ \\
cmc & $\mathbf{3 1 . 8 0 1}$ & 28.318 & 31.479 \\
dermatology & 51.825 & 50.426 & $\mathbf{5 2 . 2 6 2}$ \\
glass & $\mathbf{4 8 . 6 0 2}$ & 41.556 & 48.167 \\
haberman & 73.739 & 72.078 & $\mathbf{7 3 . 8 8 2}$ \\
heart-statlog & 74.189 & 70.133 & $\mathbf{7 5 . 4 6 7}$ \\
ionosphere & 85.733 & 70.598 & $\mathbf{8 7 . 1 1 1}$ \\
iris & 95.642 & 92.960 & $\mathbf{9 6 . 0 8 0}$ \\
monks1 & 69.271 & 56.889 & $\mathbf{7 4 . 0 8 3}$ \\
monks2 & 76.247 & 54.806 & $\mathbf{7 8 . 7 2 2}$ \\
newthyroid & 86.863 & 79.944 & $\mathbf{8 8 . 0 0 0}$ \\
page-blocks & 86.992 & 82.692 & $\mathbf{8 7 . 1 6 3}$ \\
phoneme & 79.603 & 78.735 & $\mathbf{7 9 . 8 4 7}$ \\
pima & 74.484 & 71.125 & $\mathbf{7 5 . 4 8 4}$ \\
satimage-test & 61.110 & 56.102 & $\mathbf{6 1 . 9 8 5}$ \\
tae & $\mathbf{4 6 . 0 1 3}$ & 41.961 & 44.863 \\
vehicle & 47.869 & $\mathbf{5 3 . 4 7 5}$ & 49.418 \\
wine & 81.667 & 51.667 & $\mathbf{9 0 . 2 0 0}$ \\
yeast & 32.693 & $\mathbf{3 6 . 0 6 5}$ & 32.630 \\
\hline \hline
\end{tabular}

TABLE III

AVERAGE TEST ACCURACIES IN \% FOR 25 RUNS USING CHECKING FIS FOR 20 EPOCHS. A BOLD VALUE INDICATES THE HIGHEST ACCURACY FOR THAT DATA SET.

\begin{tabular}{l|c|c|c}
\hline \hline \multicolumn{1}{c|}{ Data set } & no bags & $\begin{array}{c}\text { bags } \\
\text { majority vote }\end{array}$ & $\begin{array}{c}\text { bags } \\
\text { mean defuzzified }\end{array}$ \\
\hline balance_scale & 72.484 & 72.057 & $\mathbf{7 2 . 6 3 2}$ \\
breast & 92.764 & 81.270 & $\mathbf{9 3 . 6 1 4}$ \\
cmc & $\mathbf{3 2 . 0 0 6}$ & 28.554 & 31.796 \\
dermatology & 52.139 & 50.787 & $\mathbf{5 2 . 3 6 1}$ \\
glass & 42.284 & 40.444 & $\mathbf{5 0 . 8 3 3}$ \\
haberman & 73.736 & 72.078 & $\mathbf{7 4 . 0 0 0}$ \\
heart-statlog & 74.801 & 68.178 & $\mathbf{7 8 . 0 4 4}$ \\
ionosphere & 86.007 & 71.179 & $\mathbf{8 7 . 2 8 2}$ \\
iris & 95.365 & 91.040 & $\mathbf{9 5 . 9 2 0}$ \\
monks1 & 71.224 & 56.694 & $\mathbf{7 5 . 5 5 6}$ \\
monks2 & 82.601 & 62.083 & $\mathbf{8 4 . 8 0 6}$ \\
newthyroid & 86.279 & 80.278 & $\mathbf{8 7 . 3 3 3}$ \\
page-blocks & 88.140 & 82.323 & $\mathbf{8 8 . 7 4 3}$ \\
phoneme & 80.096 & 79.119 & $\mathbf{8 0 . 3 9 1}$ \\
pima & 74.516 & 71.125 & $\mathbf{7 5 . 5 0 0}$ \\
satimage-test & 61.403 & 56.492 & $\mathbf{6 2 . 3 0 9}$ \\
tae & $\mathbf{4 6 . 0 4 9}$ & 41.961 & 45.098 \\
vehicle & 48.326 & $\mathbf{5 3 . 8 8 7}$ & 49.773 \\
wine & 82.099 & 52.933 & $\mathbf{9 0 . 2 6 7}$ \\
yeast & 34.386 & 34.537 & $\mathbf{3 5 . 5 1 5}$ \\
\hline \hline
\end{tabular}




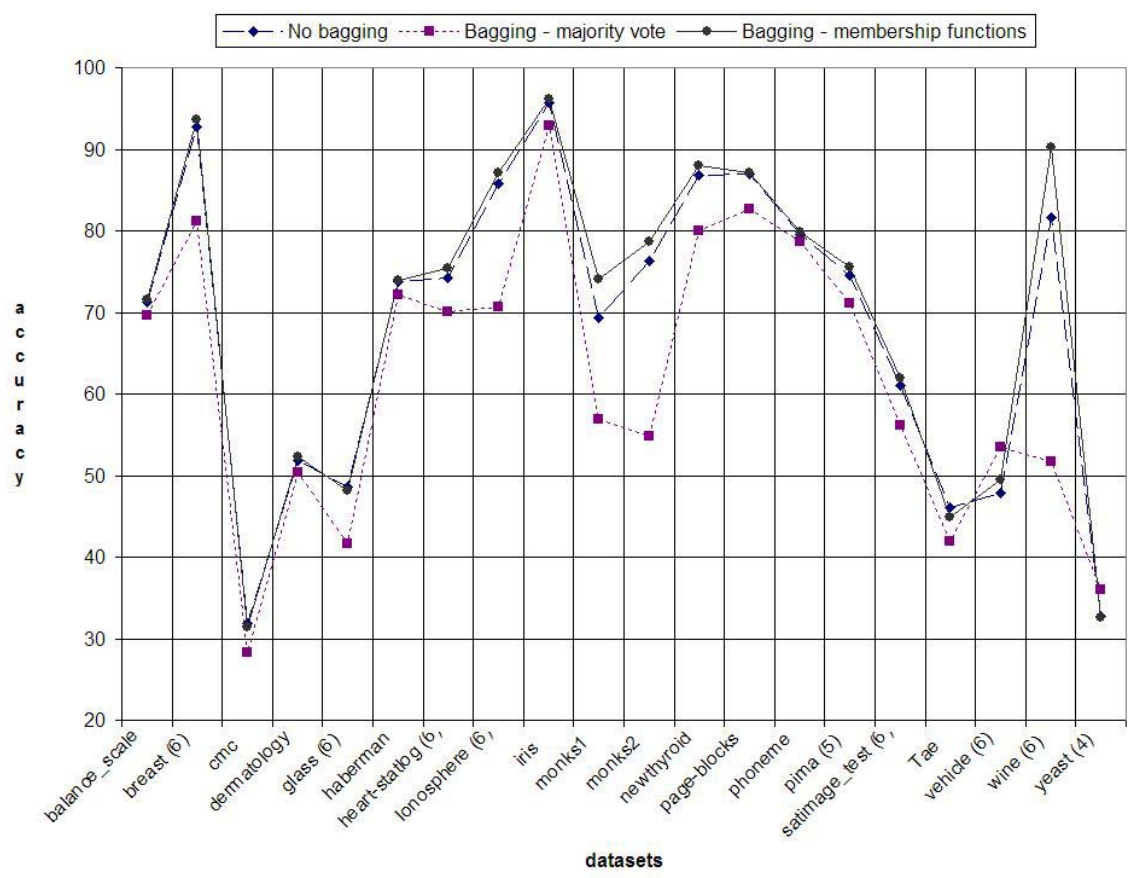

Fig. 2. Average test accuracies for 25 runs using checking FIS for 10 epochs

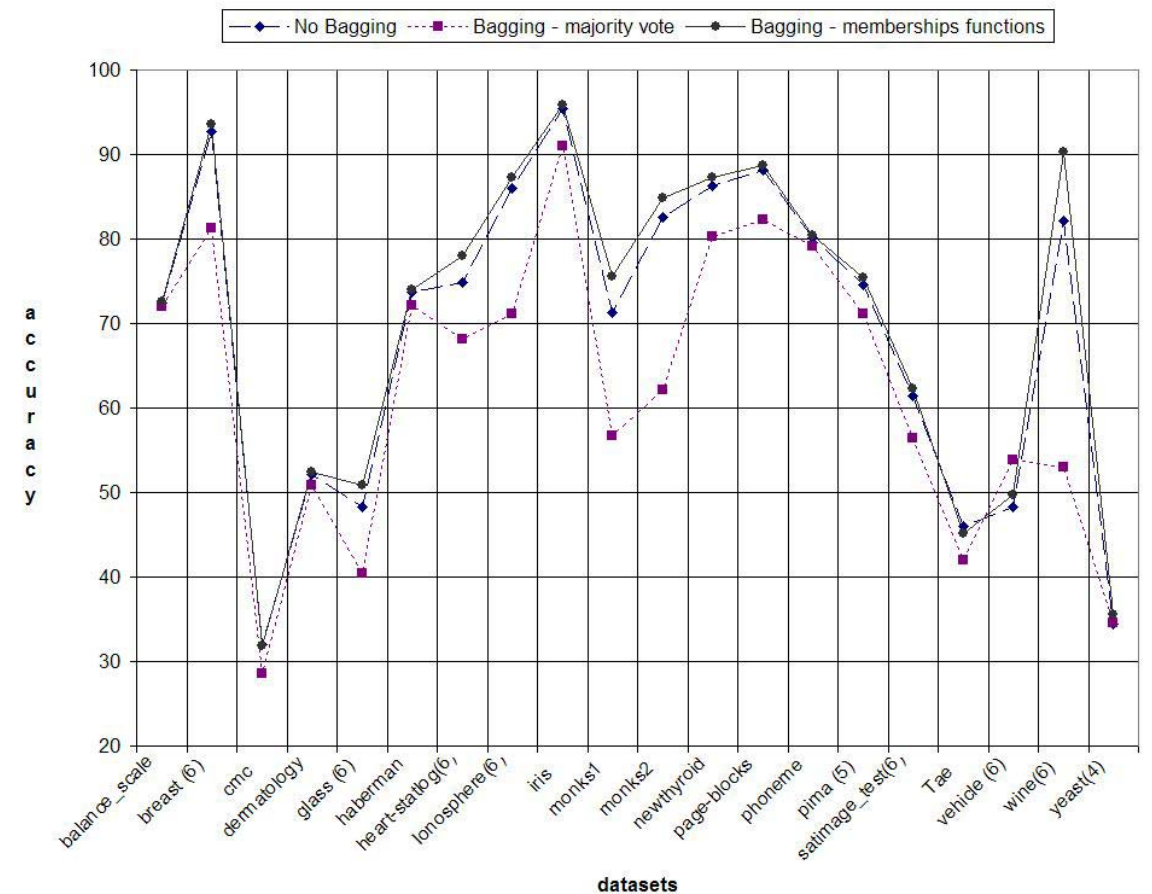

Fig. 3. Average test accuracies for 25 runs using checking FIS for 20 epochs 


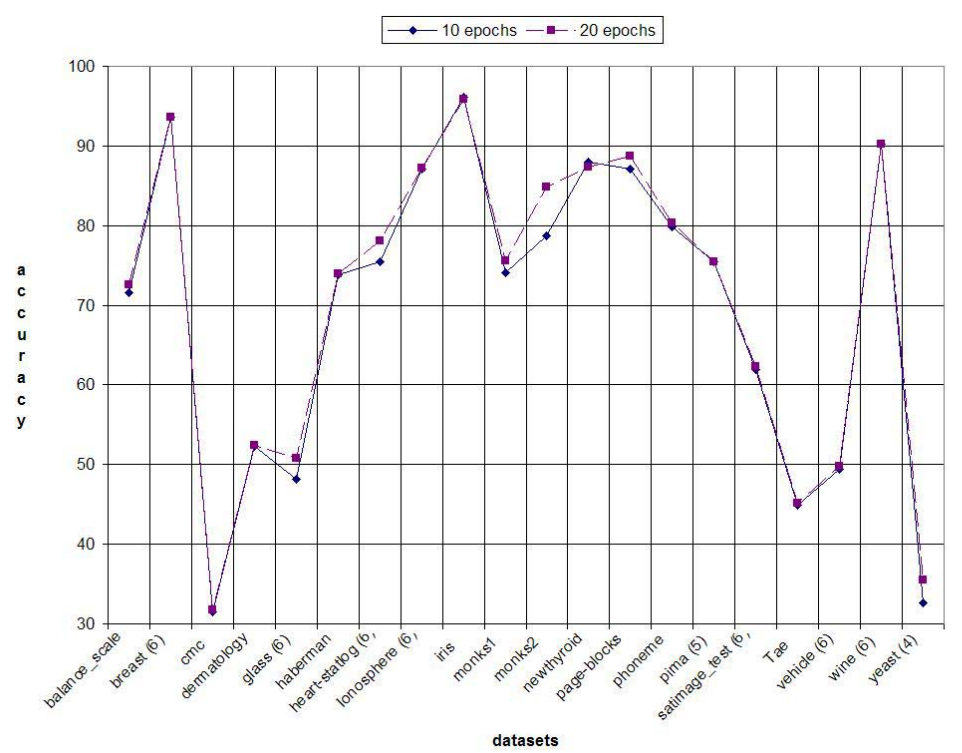

Fig. 4. Average test accuracies for 25 runs using $100 \mathrm{bags}$, and defuzzified checking FIS outputs for 10 and 20 epochs

Briefly, the Friedman test is a "non-parametric equivalent of the repeated-measures ANOVA" [11]. ANOVA is a statistical method for testing differences between the performances of classifiers measured on the same test environment with the null-hypothesis being that there is no differences between them. When the null-hypothesis is rejected, a post-hoc test follows. Holm's procedure was applied in our work. It consists of sequentially testing ordered hypotheses starting from the most significant $p$ value, if its corresponding hypothesis is rejected the procedure goes on with the next $p$ value, which is tested and so forth until a null hypothesis that cannot be rejected is found.

The Friedman-Holm test results show that using ANFIS, the bagging approach with the membership function based combination method was statistically significantly better than a single classifier, at the $95 \%$ threshold.

\section{CONCLUSIONS}

We have compared ANFIS with and without bagging to classify twenty data sets. Results were computed two different ways:

a) Ensemble class votes for each example from the defuzzified output of the FIS for each bag were individually converted to crisp class values. Then, the predicted class for each test instance was found using a majority vote of these crisp values.

b) The mean of the sum of the defuzzified FIS outputs for each bag was converted to a crisp class prediction for the ensemble.

c) Feature reduction was done via the gain ratio feature selector in Weka for all data sets with more than 6 features. Other sets of features chosen differently would result in different accuracies, but similar conclusions.
The mean defuzzified output gave the most accurate results. It is advisable not to make crisp the defuzzified outputs of each FIS before these values are combined in a vote. Otherwise, the benefit of the fuzzy membership functions is lost and lower accuracies result.

The Freidman/Holm test for determining significance of differences in accuracies for our classifier methods was performed, resulting in the conclusion bagging is statistically better than a single classifier at the $95 \%$ level.

It is interesting that fuzzy models have enough variability to benefit from an ensemble formulation. This paper shows that an ensemble of fuzzy classifiers can result in higher accuracy than a single fuzzy classifier. The next challenge is to create a single classifier that nears the accuracy of the fuzzy ensemble of classifiers, but retains the understandability of the single classifier.

\section{ACKNOWLEDGMENTS}

This research was partially supported by the Department of Energy through the ASCI PPPE Data Discovery Program, Contract number: DE-AC04-76DO00789.

This research was partially supported by the Department of Defense, National Functional Genomics Center Project, under award number DAMD 17-02-2-0051. Views and opinions of, endorsements by, the author(s) do not reflect those of the US Army or the Department of Defense.

\section{REFERENCES}

[1] R. E. Banfield et al., "A comparison of decision tree ensemble creation techniques," IEEE Transactions on Pattern Analysis and Machine Intelligence, vol. 29 No. 1, pp. 173-180, 2007.

[2] (2006) The mathworks - fuzzy logic toolbox. [Online]. Available: http://www.mathworks.ch/access/helpdesk_r13/help/ toolbox/fuzzy/fuzzy.html

[3] (2006) Uci machine learning repository. [Online]. Available: http: //www.ics.uci.edu/ mlearn/MLRepository.html 
[4] L. Breiman, "Bagging predictors," Machine Learning, vol. 24, pp. 123-140, 1996.

[5] C. A. M. Lima et al., "Fuzzy systems design via ensembles of anfis," Proceedings of the IEEE International Conference on Fuzzy Systems (FUZZ-IEEE2002), vol. 1, pp. 506-511, 2002.

[6] B. Gabrys and A. Bargiela, "General fuzzy min-max neural network for clustering and classification," IEEE Trans. on Neural Networks, vol. 11, no. 3, pp. 769-783, 2000.

[7] B. Gabrys, "Combining neuro-fuzzy classifiers for improved generalisation and reliability," in Proceedings of the Internat. Joint Conf. Neural Networks (IJCNN'2002) a part of the WCCI'2002 Congr., Honolulu, USA, May 2002, pp. 2410-2415.

[8] S. Jyh and J. Roger, "Anfis: Adaptive-network-based fuzzy inference system," IEEE Transactions on Systems, Man, and Cybernetics, vol. 23, pp. 665-685, 1993.

[9] I. H. Witten and F. Eibe, Data Mining, New Zealand, June 2005.

[10] (2006) Weka 3 - data mining with open source machine learning software in java. [Online]. Available: http://www.cs.waikato.ac.nz/ml/ weka/

[11] D. Janez, "Statistical comparisons of classifiers over multiple data sets," Machine Learning, vol. 7, pp. 1-30, 2006. 